\section{(6) OPEN ACCESS}

\title{
HIV prevalence and related risk behaviours among female partners of male injecting drugs users in Iran: results of a bio-behavioural survey, 2010
}

\author{
Abbas Alipour, ${ }^{1}$ Ali Akbar Haghdoost, ${ }^{2}$ Leily Sajadi, $^{3}$ Farzaneh Zolala $^{3}$
}

${ }^{1}$ Social Medicine Department, Mazandaran University of Medical Sciences, Sari, Iran ${ }^{2}$ Research Centre for Modelling in Health, Institute for Future Studies in Health, Kerman University of Medical Sciences, Kerman, Iran

${ }^{3}$ Regional Knowledge Hub, and WHO Collaborating Centre for HIV Surveillance, Institute for Futures Studies in Health, Kerman University of Medical Sciences, Kerman, Iran

\section{Correspondence to} Dr F Zolala, Regional Knowledge Hub for HIVIAIDS Surveillance, Kerman University of Medical Sciences, School of Health, Haftbagh-Alavi Highway, Kerman 7616913555, Iran; f.zolala@kmu.ac.ir

Received 5 May 2013 Revised 20 August 2013 Accepted 29 August 2013 Published Online First 24 September 2013

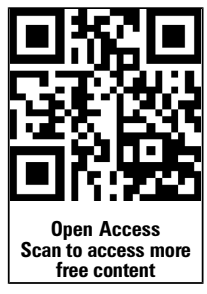

To cite: Alipour A Haghdoost AA, Sajadi L, et al. Sex Transm Infect 2013;89:iii41-iii44.

\section{ABSTRACT}

Objective Sexual partners of injecting drug users (IDUs) are at high risk of HIV infection, yet data for such populations are scarce worldwide, particularly in the Middle East and North African region. This study measured and compared the prevalence of HIV, hepatitis C (HCV), hepatitis B (HBV) and related behavioural factors in male IDUs (MIDUs), their main female sexual partners who were also injecting drug users (FIDUPs) and their main non-injecting female partners (FNIDUPs). Method Using convenience sampling, MIDUs were recruited at drop-in health centres in three cities (Tehran, Mashhad and Shiraz), who in turn recruited their main female partners. Behavioural data were collected using a standard questionnaire, and blood samples were drawn for HIV and HCV antibody testing and HBV surface antigen.

Results HIV prevalence was $9.4 \%(95 \% \mathrm{Cl} 2.96 \%$ to $26.2 \%)$ among MIDUs ( $n=226), 7.7 \%(95 \% \mathrm{Cl} 1.9 \%$ to $26.3 \%)$ among FIDUPs $(n=42)$ and $2.8 \%(95 \% \mathrm{Cl}$ $0.65 \%$ to $11.3 \%)$ among FNIDUPs $(n=184)$. HCV prevalence was $38.6 \%(95 \% \mathrm{Cl} 20.3 \%$ to $60.7 \%)$ among MIDUs, $36.6 \%(95 \% \mathrm{Cl} 13.6 \%$ to $67.9 \%)$ among FIDUPs and $8.4 \%(95 \% \mathrm{Cl} 5.67 \%$ to $12.4 \%)$ among FNIDUPs. HBV surface antigen prevalence was $3.6 \%$ (95\% Cl $1.5 \%$ to $8.3 \%), 7.3 \%$ (95\% Cl $1.9 \%$ to $24.8 \%)$ and $1.1 \%(95 \% \mathrm{Cl} 0.3 \%$ to $4.7 \%)$, respectively. Among MIDUs, $19.5 \%$ (95\% Cl $3.4 \%$ to $62.2 \%)$ had a history of sexual contact with other men. Mean age at first sexual contact in MIDUs was 19.2 years $(95 \% \mathrm{Cl}$ 18.6 to 25.2) and in FIDUPs and FNIDUPs 16.4 years (95\% Cl 14.1 to 22.1$)$ and 18.2 years $(95 \% \mathrm{Cl} 15.7$ to 23.1), respectively. FIDUPs and FNIDUPs had a higher mean number of sexual partners (other than their main partner) in the previous month than MIDUs (5.5 (95\% $\mathrm{Cl} 0$ to 14.1$)$ and $2.5(95 \% \mathrm{Cl} 1.1$ to 4$)$ vs $1.3(95 \% \mathrm{Cl}$ 0.37 to 2.2), respectively). FIDUPs tended to use drugs before or during sex with their main and casual partners more often than MIDUs (with main partner: 69\% (95\% Cl $41.5 \%$ to $87.5 \%)$ vs $54.4 \%(95 \% \mathrm{Cl} 27 \%$ to $79.4 \%)$, respectively, and with casual partners: $47.6 \%$ (95\% Cl $13.1 \%$ to $84.6 \%)$ vs $34.1 \%(95 \% \mathrm{Cl} 10 \%$ to $70.6 \%$ ), respectively); however, the differences were not statistically significant.

Conclusions Female partners of MIDUs in Iran and elsewhere are an under studied group. The high rate of HIV, HCV and HBV infection among females who are partners of MIDUs points to the necessity of appropriate injection and sexual risk reduction interventions among this group, to prevent acquisition of HIV, HCV and HBV and their onward transmission to other male partners.

\section{BACKGROUND}

Injecting drug users (IDUs) constitute a considerable number of people living with HIV/AIDS worldwide. ${ }^{1}$ Due to the common parenteral mode of transmission, IDUs are also at risk of acquiring hepatitis B virus (HBV) and hepatitis $C$ virus (HCV) infections. The sexual partners of IDUs are, in turn, at risk of HIV infection through sexual modes of transmission, and can pass the infection on to other sexual partners, or to their offspring through mother to child transmission. ${ }^{2}$ In several Asian countries, the majority of females diagnosed with HIV were either partners of men who injected drugs or were sex workers. ${ }^{3}$

There are an estimated 200 000-300 000 IDUs in Iran, the majority of whom are men. ${ }^{4}$ Of all notified HIV cases in Iran, $8.7 \%$ are among women, most of whom were infected by their sexual partners with whom they may or may not have shared injected drugs. ${ }^{5}$ However, to our best knowledge, direct measures of HIV prevalence and related factors among the partners of IDUs are rare globally, and particularly in the Middle East and North Africa (MENA) region. Establishing the prevalence of HIV and related risk behaviours among IDUs, as well as their sexual partners, is critical for planning of effective prevention and intervention programmes. As the first study of its kind in Iran, this article presents findings from a survey measuring HIV, HBV and HCV prevalence and associated risk behaviours among male IDUs (MIDUs), their main female partners who inject drugs (FIDUPs) and their main non-injecting female partners (FNIDUPs).

\section{METHODS}

MIDUs were recruited consecutively using convenience sampling from four drop-in centres (DIC) serving IDUs in three large cities in Iran in 2010 (one in Tehran, one in Mashhad and two in Shiraz) and asked to participate in a bio-behavioural survey which included bringing in their main female sexual partners. DIC are supported by the Iranian government, and serve subgroups with high risk behaviours who inject drugs and who have high risk sexual behaviours. DIC provide services and support, such as methadone therapy and counselling.

Eligible MIDUs were $\geq 18$ years old, had a history of at least one drug injection in the previous year and had at least one female partner within the past 6 months. Eligible MIDUs who had a sexual partner in the past 6 months were asked if their 
partner had also consented to participate, and a time was set for them to come together with their main female partner for interview. Of the MIDUs, almost $80 \%$ attended with their partner to participate in the study. Based on the lifetime history of drug injection, partners were categorised as FIDUPs or FNIDUPs. After obtaining verbal consent, participants were interviewed by a trained professional using a standard survey questionnaire covering demographics, drug use and injection practices, and sexual risk behaviours. The partners were interviewed separately by a same gender interviewer. Dried blood spots were collected on S\&S 903 filter paper and a whole blood test was carried out on each participant after obtaining separate consent for serological testing for HIV, HCV antibodies and HBV surface antigen. All testing used commercial assays for HIV (ELISA-Gene screen, BIO-RAD and confirmed with western blot-MP Diagnostic), HCV (ELISA- Dia Pro and confirmed with western blot- RIBA) and HBV (ELISA-Dia Pro and confirmed with ELISA-Gene screen, BIO-RAD). ${ }^{6}$ Participants were given an incentive of US $\$ 4$ to compensate them for their participation. Results for all participants were disclosed at the DIC, with referral to care and treatment as appropriate. The study was approved by the ethics committee at Kerman University of Medical Sciences.

An a priori sample size of 230 MIDUs was calculated based on the detection of a minimum of $5 \%$ prevalence of HIV infection in their partners within a $\pm 3 \%$ margin of error, with $95 \%$ CI. The data were weighted and analysed using survey analysis commands in Stata V.10 to take into account clustering at the level of the sites.

\section{RESULTS}

We recruited 226 MIDUs (mean age 37.0 years; 95\% CI 34 to 40.2) and 226 main female partners (mean age 34.1 years; $95 \%$ CI 32.5 to 35.4) (table 1). Most MIDUs and FNIDUPs were married $(61.0 \%$; $95 \%$ CI $48.7 \%$ to $71.4 \%)$ and $66.7 \%$ (95\%

Table 1 Description of participants: male injection drug users and their main female partners, including injecting drug user partners and non-injecting drug user partners (Iran 2011)

\begin{tabular}{|c|c|c|c|c|c|}
\hline & \multirow{2}{*}{$\begin{array}{l}\text { MIDUs } \\
(\mathrm{n}=226)^{*}\end{array}$} & \multicolumn{2}{|l|}{ Main female partners } & \multicolumn{2}{|c|}{$\underline{p \text { Valuet }}$} \\
\hline & & FIDUPs $(n=42(18.6 \%))$ & FNIDUPs $(n=184(81.4 \%))$ & A & B \\
\hline \multicolumn{6}{|l|}{ Demographic characteristics } \\
\hline Age (years) $($ mean $\pm S D)$ & $37.0 \pm 1.1$ & $33 \pm 1$ & $34.1 \pm 0.7$ & 0.43 & 0.07 \\
\hline \multicolumn{6}{|l|}{ Marital status (n (\%)) } \\
\hline Single & $30(13.3)$ & $4(9.5)$ & $9(4.9)$ & & 0.08 \\
\hline Married & $137(61.0)$ & $11(26.2)$ & $122(66.7)$ & & \\
\hline Divorced & $16(7.1)$ & $7(16.7)$ & $19(10.4)$ & & \\
\hline Temporary marriage (sigheh) & $42(18.6)$ & $17(40.5)$ & $29(15.8)$ & & \\
\hline Widowed & $1(0.4)$ & $3(7.1)$ & $4(2.2)$ & & \\
\hline \multicolumn{6}{|l|}{ Education (n (\%)) } \\
\hline None & $23(10.2)$ & $6(14.3)$ & $41(22.4)$ & 0.31 & 0.31 \\
\hline Primary & $146(64.6)$ & $24(57.1)$ & $108(59.0)$ & & \\
\hline High school & $57(25.2)$ & $12(28.6)$ & $34(18.6)$ & & \\
\hline \multicolumn{6}{|l|}{ Drug related risks (n (\%)) } \\
\hline History of drug abuse (n (\%)) & $226(100)$ & $42(100)$ & $88(47.8)$ & & \\
\hline Age at first drug use (years) (mean $\pm S D$ ) & $18.5 \pm 0.4$ & $20.3 \pm 1.2$ & $21.8 \pm 1.5$ & 0.33 & 0.21 \\
\hline Age at first drug injection (years) (mean $\pm S D$ ) & $27.0 \pm 1.2$ & $27.4 \pm 1$ & - & - & 0.64 \\
\hline History of high risk drug injection (n (\%)) & $84(39.1)$ & $22(55.0)$ & - & - & 0.23 \\
\hline \multicolumn{6}{|l|}{ Sex related risks } \\
\hline Age at first sexual contact (years) (mean $\pm S D$ ) & $19.2 \pm 3.9$ & $16.4 \pm 2.4$ & $18.2 \pm 4.5$ & & \\
\hline No of sexual acts with main partner in previous month (mean \pm SD) & $5.4 \pm 1.1$ & $5.3 \pm 0.85$ & $4.4 \pm 1.2$ & 0.43 & 0.89 \\
\hline Having sex with casual partner (n (\%)) & $157(72.7)$ & $33(78.6)$ & 77 (41.8) & 0.06 & 0.42 \\
\hline No of sexual acts with casual partner in previous month (mean $\pm S D$ ) & $1.7 \pm 0.8$ & $4.8 \pm 1$ & $13 \pm 5.9$ & 0.29 & 0.10 \\
\hline No of sexual partners in last 6 months (excluding main partner) $($ mean $\pm S D)$ & $1.3 \pm 0.3$ & $2.5 \pm 0.5$ & $5.5 \pm 4.2$ & 0.47 & 0.36 \\
\hline Condom use with main partner in past 6 months (overall) (n (\%)) & $126(55.8)$ & $24(57.1)$ & $90(49.5)$ & 0.29 & 0.77 \\
\hline Condom use with casual partner in past 6 months (overall) (n (\%)) & $58(25.7)$ & $18(42.9)$ & $45(24.5)$ & 0.23 & 0.16 \\
\hline Drug use before or during last sex with main partner $(\mathrm{n}(\%))$ & $123(54.4)$ & $29(69.0)$ & $54(29.7)$ & 0.03 & 0.44 \\
\hline Drug use before or during last sex with casual partner (n (\%)) & $77(34.1)$ & $20(47.6)$ & $21(11.4)$ & 0.02 & 0.38 \\
\hline History of genital ulcer in the previous year ( $\mathrm{n}(\%))$ & $24(10.7)$ & $8(19.0)$ & $37(20.8)$ & 0.62 & 0.04 \\
\hline History of genital discharge in the previous year (n (\%)) & $20(8.9)$ & $24(58.5)$ & $90(49.7)$ & 0.14 & 0.01 \\
\hline $\begin{array}{l}\text { Correctly identified ways of preventing sexual transmission and rejected major } \\
\text { misconceptions about HIV transmission }(\mathrm{n}(\%))\end{array}$ & $40(22.5)$ & $8(21.6)$ & $40(33.1)$ & 0.13 & 0.77 \\
\hline \multicolumn{6}{|l|}{ Serologic status and prior testing } \\
\hline HIV antibody positive (n (\%)) & $21(9.4)$ & $3(7.7)$ & $5(2.8)$ & 0.11 & 0.63 \\
\hline HCV antibody positive (n (\%)) & $86(38.6)$ & $15(36.6)$ & $15(8.4)$ & 0.01 & 0.90 \\
\hline HBV antigen positive ( $\mathrm{n}(\%))$ & $8(3.6)$ & $3(7.3)$ & $2(1.1)$ & 0.03 & 0.37 \\
\hline Tested for HIV in the past 12 months and received result $(\mathrm{n}(\%))$ & $30(13.3)$ & $7(16.7)$ & $26(14.1)$ & 0.68 & 0.21 \\
\hline
\end{tabular}

*Male injecting drug users.

†A: MIDUs versus main heterosexual partners; A: MIDUs versus FNIDUPs. B: MIDUs versus FIDUPs.

HBV, hepatitis B virus; HCV, hepatitis C virus; MIDUs, male injecting drug users; FIDUPs, main female partners of male injecting drug users; FNIDUPs, non-injecting female partners of male injecting drug users. 
CI $47.8 \%$ to $81.4 \%$ ), respectively, while $26.2 \%$ (95\% CI $15.7 \%$ to $40.3 \%$ ) of FIDUPs were married and $40.5 \%$ (95\% CI $21.2 \%$ to $63.2 \%$ ) were in temporary marriages (sigheh). The majority of participants in all groups were educated to no higher than secondary school level.

Of the FNIDUs, $47.8 \%$ had used drugs and $18.6 \%$ (95\% CI $12.6 \%$ to $26.5 \%$ ) had a prior history of drug injection use. MIDUs, FIDUPs and FNIDUPs who used drugs also reported using crystal, heroin and opium. MIDUs reported starting drug use at a younger age than their partners, with a mean age at first use of 18.5 years (95\% CI 17.5 to 19.6 ) and 21.3 years $(95 \%$ CI 17.9 to 24.8), respectively. MIDUs reported starting to inject drugs at an age similar to FIDUPs (27.9 years; 95\% CI 24.8 to 31.2 ) vs 27.4 years (95\% CI 24.7 to 30 ), respectively). Sharing needles was more common among FIDUPs than MIDUs, reported by $55.0 \%$ (95\% CI $39.5 \%$ to $70.6 \%$ ) compared with $39.1 \%$ (95\% CI $30 \%$ to $50 \%$ ), respectively.

Table 1 shows the multiple sexual risk behaviours reported by the three groups. Women were younger at first sexual contact than their MIDU partners, at 16.4 years (95\% CI 14.1 to 22.1 ) for FIDUPs compared with 18.2 years (95\% CI 15.7 to 23.1 ) for FNIDUPs and 19.2 years (95\% CI 18.7 to 25.2) for MIDUs. Most MIDUs $(72.7 \%$; 95\% CI $46.5 \%$ to $85.6 \%$ ) and FIDUPs (78.6\%; 95\% CI $44.5 \%$ to $94.4 \%$ ) reported casual partners (other than the main one they came with); FNIDUPs had a high mean number of partners in the past 6 months $(5.5$ (95\% CI 0 to 14.1) casual partners). Reported condom use and drug use before or during sex tended to be higher with the main partner compared with casual partners. Injection and non-injection drug use before or during sex was common among MIDUs and FIDUPs (54.4\% (95\% CI $27 \%$ to $79.4 \%$ ) and $69.0 \%$ (95\% CI $41.5 \%$ to $87.5 \%$ ), respectively with main partners, and $34.1 \%$ (95\% CI $10 \%$ to $70.6 \%$ ) and $47.6 \%$ (95\% CI $13.1 \%$ to $84.6 \%$ ), respectively with casual partners) and yet a substantial fraction of the FNIDUPs also used drugs with sex with their main partners $(29.7 \%$; $95 \%$ CI $18.6 \%$ to $43.8 \%)$ and with casual partners $(11.4 \%$; $95 \%$ CI $5.1 \%$ to $23.8 \%)$. Symptoms of sexually transmitted infections, such as genital ulcers and genital discharge, were also fairly common for both types of female partners. MIDUs tended to have a lower level of comprehensive knowledge of HIV/AIDS than their female partners $(22.5 \%$; $95 \%$ CI $2.81 \%$ to $74.4 \%$ vs $30.4 \%$; $95 \%$ CI $7.4 \%$ to $70.3 \%$, respectively) although the difference was not statistically significant.

The prevalence of HIV and HCV in MIDUs tended to be higher than in FIDUPs and FNIDUPs. HIV prevalence was $9.4 \%$ (95\% CI $2.96 \%$ to $26.2 \%$ ) in MIDUs compared with $7.7 \%$ (95\% CI $1.9 \%$ to $26.3 \%$ ) and $2.8 \%$ (95\% CI $0.65 \%$ to $11.3 \%$ ) in FIDUPs and FNIDUPs, respectively. HCV prevalence was $38.6 \%(95 \%$ CI $20.3 \%$ to $60.7 \%)$ in MIDUs compared with $36.6 \%$ (95\% CI $13.6 \%$ to $67.9 \%)$ and $8.4 \%(95 \%$ CI $5.67 \%$ to $12.4 \%$ ) in FIDUPs and FNIDUPs, respectively. The prevalence of HBV was lower in MIDUs than in FIDUPs, but higher than in female FNIDUPs (3.6\% (95\% CI 1.5\% to $8.3 \%$ ) vs $7.3 \%$ (95\% CI $1.9 \%$ to $24.8 \%$ ) and $1.1 \%$ (95\% CI $0.26 \%$ to $4.7 \%$ ), respectively). Fewer than one in five in any group had been tested for HIV in the past 12 months and received their results $(13.3 \%$ (95\% CI $6.2 \%$ to $26.2 \%$ ) of MIDUs, $16.7 \%$ (95\% CI $5.3 \%$ to $41.5 \%$ ) of FIDUPs and $14.1 \%$ (95\% CI $5.9 \%$ to $30.4 \%$ ) of FNIDUPs). Among the participants, two couples were HIV concordant positive-that is, both the man and woman were HIV positive. One of these couples involved an FNIDUP and the other involved an FIDUP.

\section{DISCUSSION}

To our knowledge, this study is one of only a few in the MENA region that has measured the level of HIV infection among female sexual partners of MIDUs-a key group at risk of HIV infection that has been largely neglected or has been beyond the reach of researchers in this region. Through their MIDU partners, we obtained rare samples of female partners of IDUs. Our results showed that HIV prevalence was higher among FIDUPs (7.7\%) compared with FNIDUPs $(2.8 \%)$, mainly because of the dual risk of unsafe injection and unprotected sex. Moreover, $\mathrm{HCV}$ affected more than one-third of MIDUs and FIDUPs and $8.4 \%$ of FNIDUPs. These results are of great concern in Iran, as the per capita prevalence of injecting drug use is quite high. ${ }^{4}$ In the province of Kerman (not the study setting) for example, it was estimated that the prevalence of injecting drug use among males aged 18-45 years was $1.2 \% .^{7}$ Substantial levels of HIV, $\mathrm{HCV}$ and HBV infection among IDUs have already been documented in Iran, with a prevalence of approximately $10.7 \%$, $34.5 \%$ and $50.7 \%$, respectively. ${ }^{8}$

The relevance of partners of IDUs to HIV dynamics was illustrated in a recent modelling study that estimated HIV incidence by mode of exposure. According to the study, about 1000 new HIV infections per year occur among the partners of IDUs in Iran-the second highest level of HIV incidence after IDUs. ${ }^{9}$

Our results provide a rare insight into the sexual risks of women within the sexual networks of MIDUs in Iran. In our survey, many women and men reported having casual sexual partners in addition to their main partner. We hypothesise that a number of participants may have been sex workers during the past month. Due to the situation in Iran, where potentially severe penalties exist for sex workers, we chose not to ask this question directly. But if this is true, the overlap of these different key populations can create opportunities for bridging HIV infection to other subpopulations, such as female sex workers. Temporary marriage (sigheh, which is a type of marriage with a flexible but predetermined duration), may also facilitate HIV transmission through the formation of multiple and potentially concurrent partnerships.

Taking into account the stigma that exists around high risk behaviours in Iran, some of our self-reported measures may have been affected by underreporting bias. Moreover, this was a facility based study and accordingly prone to selection bias and may not be generalisable. Due to the nature of the affected populations, our method of recruitment was the most feasible option to conduct this study.

Female partners of IDUs have received inadequate attention with respect to care and prevention programmes in Iran, and likely elsewhere in the MENA region. This is of concern not only because of the risk of HIV acquisition, but also because of the risk of mother to child transmission. Our study calls for further research in the region to understand the vulnerability of spouses and other partners of MIDUs and to explore avenues by which this vulnerability can be addressed. HIV surveillance programmes for female partners of IDUs are needed. Given that the levels of HIV infection in the general population at large are still very low, general population based interventions may not be cost effective and may be confronted by multiple logistical barriers. ${ }^{10}$ However, people who use illicit drugs could be targeted for specific interventions. ${ }^{11}$

Targeted interventions may have the potential for more success, but access to women at risk may be difficult. Novel approaches to HIV prevention among these women, which are appropriate for the MENA context, are urgently needed, with 
the increasing evidence for emerging and established HIV epidemics in MENA. ${ }^{12}$ Our success in reaching and recruiting these women through their MIDU partners offers a promising starting point, and this approach of recruitment of female partners could be applied to the provision of appropriate interventions.

\section{Key messages}

- High levels of HIV and hepatitis C virus infection are found among injecting drug users and their main female sexual partners.

- Injecting and sexual risky behaviours are common among injecting drug users and their main female sexual partners.

- Female sexual partners of injecting drug users are a key vulnerable population at risk of HIV infection and may play a role in bridging HIV infection to other population groups.

\section{Handling editor Jackie A Cassell}

Acknowledgements We gratefully acknowledge the technical help and support of the United Nations Office for Drugs and Crimes (Mr Antonio de Leo, Dr Setareh Mohsenifar and Mr Kamran Khaki), UNAIDS, with special thanks to Dr Doroudi, the Ministry of Health (Dr Abbas Sedaghat and Dr Nooshin Fahimfar) and the Pasteur Institute in Iran (Dr Mohammad Reza Aghasadeghi, Mr Farhang Vahabpour and Dr Mehdi Sadat), and Dr Armita Shahesmaeili and Ms Afsar Bouzarjomehri, as well as all the interviewers and participants. We would also like to thank Dr Soodabeh Navadeh, Dr Ali Mirzazadeh and Dr Ehsan Mostafavi for their efforts in preparing and finalising the proposal and questionnaires.

Contributors AA and LS contributed to the concept and design of the study, monitoring of data collection and quality, analysis and interpretation of the data. FZ was involved in interpreting the results, drafting the manuscript and revising it based on reviewer comments. AAH made substantial contributions to the concept, design, analysis and interpretation of the findings.

Funding This work was funded by the United Nations Office for Drugs and Crimes, Iran Country Office, Under UNAIDS Programme Acceleration Fund (PAF) 2009/2010 (grant number: $\mathrm{IC/10/26)}$.

\section{Competing interests None.}

Ethics approval The protocol was approved by the ethics committee of Kerman University of Medical Sciences.
Provenance and peer review Commissioned; externally peer reviewed.

Open Access This is an Open Access article distributed in accordance with the Creative Commons Attribution Non Commercial (CC BY-NC 3.0) license, which permits others to distribute, remix, adapt, build upon this work non-commercially, and license their derivative works on different terms, provided the original work is properly cited and the use is non-commercial. See: http://creativecommons.org/ licenses/by-nc/3.0/

\section{REFERENCES}

1 Mathers BM, Degenhardt L, Phillips B, et al. Global epidemiology of injecting drug use and HIV among people who inject drugs: a systematic review. Lancet 2008; $372: 1733-45$

2 Mills HL, White E, Colijn C, et al. HIV transmission from drug injectors to partners who do not inject, and beyond: modelling the potential for a generalized heterosexual epidemic in St. Petersburg, Russia. Drug Alcohol Depen Published Online First: 18 May 2013. doi:10.1016/j.drugalcdep.2013.04.028.

3 HIV transmission in intimate partner relationships in Asia. UNAIDS 2009 [cited 2011], 2009.

4 Alavi SM, Behdad F. Seroprevalence study of hepatitis C and hepatitis B virus among hospitalized intravenous drug users in Ahvaz, Iran (2002-2006). Hepat Mon 2010;10:101.

5 Islamic Republic of Iran *AIDS Progress Report On Monitoring of the United Nations General Assembly Special Session on HIV and AIDS. 2012 2013. http://www.unaids. org/en/dataanalysis/knowyourresponse/countryprogressreports/2012countries/RIran \%20AIDS\%20Progress\%20Report\%202012\%20English\%20final1_1.pdf

6 Maity S, Nandi S, Biswas S, et al. Performance and diagnostic usefulness of commercially available immunosorbent assay and rapid kits for detection of HIV HBV and HCV in India. Virol I 2012:9:290.

7 Shokoohi M, Baneshi MR, Haghdoost A-a. Size estimation of groups at high risk of HIV/AIDS using network scale up in Kerman, Iran. Inter J Prev Med 2012;3:471.

8 Rahimi-Movaghar A, Razaghi EM, Sahimi-Izadian E, et al. HIV, hepatitis C virus, and hepatitis B virus co-infections among injecting drug users in Tehran, Iran. Int J Infect Dis 2010;14:21.

9 Nasirian M, Doroudi F, Gooya MM, et al. Modeling of human immunodeficiency virus modes of transmission in iran. JRHS 2012:12:81-7.

10 Abu-Raddad L, Sgaier SK, Mumtaz GR. HIV response in the Middle East and North Africa: an epidemic and its policy dilemmas. In: Smith RA.ed. Global HIV/AIDS activism, politics, and policy: persistent challenges and emerging issues. Washington, DC: Praeger Publishers, 2013:143-68.

11 Belani H, Chorba T, Fletcher F, et al. Integrated Prevention Services for HIV Infection, Viral Hepatitis, Sexually Transmitted Diseases, and Tuberculosis for Persons Who Use Drugs Illicitly: Summary Guidance from CDC and the U.S. Department of Health and Human Services, 2012. http://www.cdc.gov/mmwr/ preview/mmwrhtml/rr6105a1.htm

12 Abu-Raddad LJ, Akala FA, Semini I. Characterizing the HIVIAIDS epidemic in the Middle East and North Africa: time for strategic action. World Bank Publications, 2010. 